\title{
EU-Kommission will Mobilität erleichtern Berufsanerkennungen im europäischen Binnenmarkt
}

Die Europäische Kommission in Brüssel hat Mitte Dezember 2011 ihre Pläne für eine schnellere und einfachere Anerkennung der Berufsqualifikationen von rund 800 reglementierten Berufen vorgelegt. Der Vorschlag sieht unter anderem vor, dass einzelne Branchen künftig freiwillig europäische Berufsausweise einführen können, die die Abwicklung des Anerkennungsverfahrens erleichtern sollen. Die Europäische Kommission will darüber hinaus die Pflegeausbildung EU-weit auf 12 Jahre festlegen. Bei der Bundesregierung und Interessenvertretern stößt die Idee auf Kritik. Sie fürchten, dass dies den Fachkräftemangel in der Pflege weiter verschärfen könnte. Der Legislativvorschlag der EU-Kommission muss noch vom Europäischen Parlament und den Regierungen beraten werden.

Die Europäische Kommission will die grenzüberschreitende Mobilität von Arbeitnehmern verbessern. Dies gilt auch für Fachkräfte aus dem Gesundheitswesen, wie Apotheker, Ärzte, Hebammen, Krankenschwestern und -pfleger sowie Zahnärzte. Denn Bereichen wie der Medizin und der Pflege droht in fast allen EUStaaten ein zunehmender Mangel an qualifiziertem Personal, mit dem sie die Herausforderungen durch den demographischen Wandel bewältigen können. Die Brüsseler Binnenmarktexperten schätzen, dass derzeit innerhalb der EU bereits etwa eine Million Fachkräfte fehlen. Diese Zahl könnte sich in den kommenden Jah-

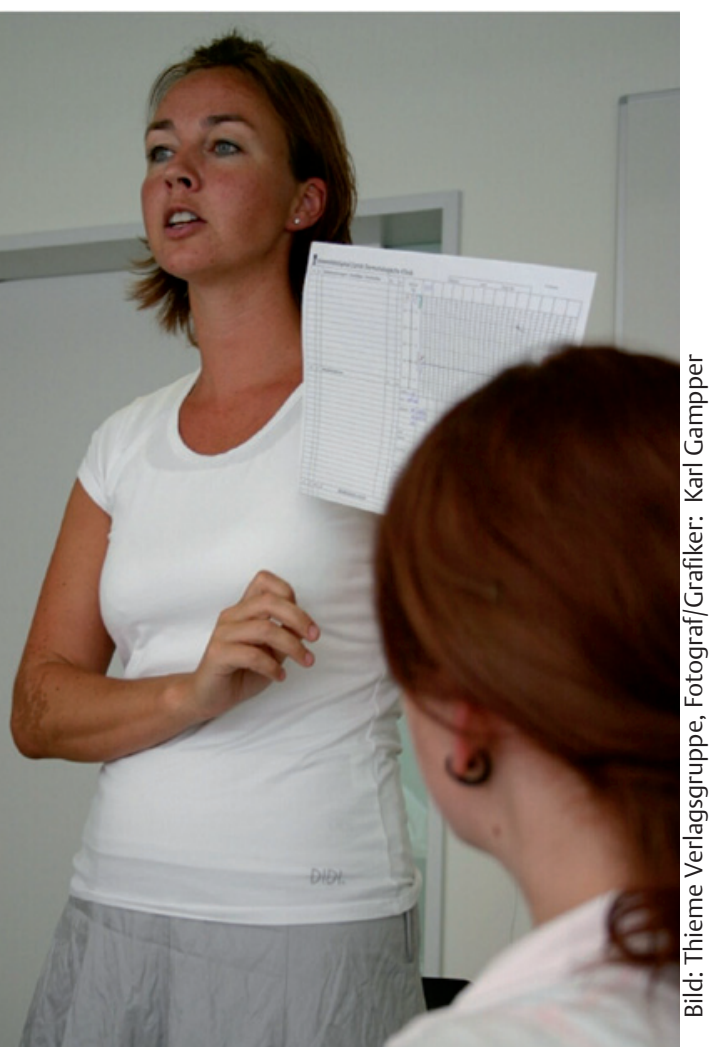

ren verdoppeln, wenn die Regierungen nicht mit geeigneten Maßnahmen gegensteuern, prophezeit die Europäische Kommission.

Zu diesem Zweck hat die Brüsseler Behörde die Richtlinie über die Anerkennung von Berufsqualifikationen (2005/36/EG) neu gefasst und ihre Pläne Mitte Dezember 2011 in Brüssel offiziell präsentiert. Die zuletzt im Jahr 2005 überarbeitete Direktive regelt, welche Voraussetzungen erfüllt sein müssen, um eine in einem Mitgliedstaat erworbene Berufsqualifikation in einem anderen Mitgliedstaat anzuerkennen.

Für Ärzte, Pflegepersonal und andere reglementierte Gesundheitsberufe in der EU gilt das Prinzip der automatischen Berufsanerkennung auf der Grundlage harmonisierter Mindestausbildungsanforderungen, anders als beispielsweise in den Bereichen Handwerk, Architektur, Industrie und Handel. Hier ist jeweils eine aufwändigere gegenseitige Anerkennung erforderlich.

Zu den Voraussetzungen für die Qualifikationen auf europäischer Ebene gehört eine medizinische Grundausbildung von mindestens 6 Jahren, beziehungsweise 5500 Stunden theoretischem und praktischem Unterricht an einer Universität. Auch für die Dauer und Inhalte der Weiterbildung der Fachdisziplinen gelten einheitliche Mindestanforderungen. Ferner kann der Aufnahmestaat Nachweise über die Zuverlässigkeit, Straffreiheit und Gesundheit des Arztes verlangen.

Anerkennungsverfahren weiter vereinfachen

Mit der Neufassung der EU-Regeln will die Kommission die Anerkennungsver- fahren weiter vereinfachen und beschleunigen. „Es wird in Zukunft weniger darum gehen, dass Bürger in einem Aufnahmemitgliedstaat Zugang zu einem Beruf erhalten, sondern eher darum, dass die Mitgliedstaaten qualifizierte Berufstätige zum Kommen ermutigen“, betonte Jürgen Tiedje, Leiter des Referats Freizügigkeit von Fachkräften bei der zuständigen Generaldirektion der Kommission auf einem Fachsymposium in Brüssel. Bislang hält sich die Motivation bei Ärzten, eine Zeitlang oder dauerhaft im europäischen Ausland zu arbeiten, noch in Grenzen. Zwischen 2007 und 2010 haben nach Angaben von Tiedje lediglich 26000 Ärzte in der EU eine automatische Anerkennung beantragt. Die Praxis habe zudem gezeigt, dass die Anerkennungsverfahren nicht immer reibungslos verlaufen, sei es aufgrund falscher Gesetzesauslegungen oder wegen Missverständnissen über die Qualifikation eines Arztes.

Für die grenzüberschreitende Mobilität von Ärzten gibt es verschiedene Ursachen. Aus Deutschland wandern Ärzte vor allem aus, weil sie mit den Arbeitsbedingungen unzufrieden sind. Gehaltssorgen dürften eine eher untergeordnete Rolle spielen. Denn das verfügbare kaufkraftbereinigte Einkommen von Krankenhausärzten in Deutschland liegt einer Studie des Deutschen Krankenhausinstituts zufolge im europäischen Vergleich im oberen Bereich. „Lediglich Länder wie Großbritannien, die Schweiz und die Niederlande, die seit Jahrzehnten für den eigenen Bedarf zu wenig Fachärzte weiterbilden, sind gezwungen, aufgrund der selbstinduzierten Knappheit noch höhere Gehälter für Fachärzte zu zahlen“, so der Hauptgeschäftsführer der Deutschen Krankenhausgesellschaft (DKG), Georg Baum.

Ärzte aus Österreich wiederum zieht es ins Ausland, weil sie in ihrer Heimat aufgrund hoher Absolventenzahlen geringe Aussichten auf eine Assistenzarztstelle haben. Polnische Ärzte werden durch den besseren Verdienst in anderen Ländern angezogen. Schweden benötigt ausländische Kräfte wegen seines hohen Personalbedarfs. In Großbritannien machen vor allem die Reformen im Gesundheits- 
wesen eine Rekrutierung ausländischer Ärzte erforderlich.

Spitzenreiter bei der Rekrutierung ausländischer Ärzte in der EU ist Großbritannien mit 37\% am gesamten Ärzteaufkommen. Der Ausländeranteil bei den in Deutschland gemeldeten Ärzten betrug nach einer Statistik der Bundesärztekammer (BÄK) 2010 dagegen nur 7,9\%. Dabei stammten $73 \%$ der ausländischen Ärzte aus EU-Staaten, insbesondere aus Österreich, Griechenland, Polen und Rumänien. 2010 wanderten zugleich 3241 Ärzte aus Deutschland aus. Zielländer in der EU sind vor allem Großbritannien, Österreich und Schweden.

Ein europäischer Berufsausweis Im Mittelpunkt der Überlegungen von Binnenmarktkommissar Michel Barnier bei der Neufassung der Anerkennungsvoraussetzungen steht die Einführung eines europäischen Berufsausweises. Dieser soll u.a. Auskunft darüber geben, welche Ausbildung der Berufstätige im Herkunftsmitgliedstaat durchlaufen hat. Die EU-Kommission erhofft sich von den Ausweisen einen schnelleren Informationsaustausch und somit eine Verkürzung der Fristen für die Anerkennung. Nach den Vorstellungen der Behörde sollten 2 Wochen für die Überprüfung der Voraussetzungen statt der bislang üblichen 3 Monate ausreichen.

Die Kommission will zugleich eine Genehmigungsfiktion einführen. Dies würde bedeuten, dass eine Anerkennung als erteilt gilt, wenn die zuständige Behörde innerhalb der vorgeschriebenen Frist keinen gegenteiligen Bescheid gibt. Ein Arzt dürfte somit nach Ablauf der 14-Tagesfrist rechtmäßig im Aufnahmestaat praktizieren.

Die Behörde will die Ausweise jedoch nicht verpflichtend vorschreiben. Vielmehr sollen die einzelnen Branchen selbst über die Einführung entscheiden. Das Europäische Parlament (EP) hat sich bereits ebenfalls mit großer Mehrheit für eine freiwillige Einführung ausgesprochen. In jedem Mitgliedstaat soll nach den Vorstellungen der Kommission zudem nur eine zentrale Stelle das Verfahren zur Erteilung des Berufsausweises durchführen. Die Behörde will die Stellen ferner dazu verpflichten, alle erforderlichen Angaben auf einer gemeinsamen Internetplattform bereitzustellen.

Die Vorteile liegen nach Ansicht von Klaus-Dieter Sohn vom Centrum für Eu- ropäische Politik auf der Hand: „Migrationswillige Berufstätige müssen sich nicht mehr einem Anerkennungsverfahren in fremder Sprache im Aufnahmemitgliedstaat stellen, sondern können alle Formalitäten in ihrer Heimat abwickeln.“ Die Arbeitgeber - zum Beispiel Krankenhäuser - könnten ferner die Qualifikation eines Bewerbers problemlos auf einer standardisierten Informationsbasis anhand des Berufsausweises feststellen.

Die DKG warnt unterdessen davor, Arbeitskräfte von einem Staat in einen anderen verschieben zu wollen. Hierdurch lasse sich das grundlegende Problem des Fachkräftemangels nicht lösen, so Marc Schreiner, Abteilungsleiter Europäische und Internationale Politik bei der DKG.

Krankenschwester - nur noch mit Abitur?

Ein weiteres Element der Neuregelungen sind geänderte Mindestansprüche bei bestimmten Berufsausbildungen. Die EUKommission regt beispielsweise an, die schulischen Voraussetzungen zur Krankenschwester beziehungsweise zum Krankenpfleger und zur Hebamme EUweit auf 12 Jahre festzulegen, da sich, so die Begründung, diese inzwischen sehr viel größeren Herausforderungen bei der Gesundheitsversorgung stellen müssten. Dies stößt hierzulande jedoch auf Widerstand bei der Bundesregierung und Interessenverbänden. Sie fürchten, dass eine längere Schulbildung zulasten der ohnehin rückläufigen Zahl an Pflegekräften gehen könnte. Im Bundestag wird der Vorschlag ebenfalls kritisch gesehen. So hat sich der Gesundheitsausschuss des Deutschen Bundestages in einem Fraktionsübergreifenden Entschließungsantrag zur Überarbeitung der EU-Richtlinie zu den Berufsqualifikationen mit überwiegender Mehrheit dagegen ausgesprochen, die Anforderungen zur Pflegeausbildung durch die EU von 10 auf 12 Schuljahre zu erhöhen. „Die Ausbildung zu den Pflegeberufen in Deutschland hat sich bewährt und ist anerkannt“, sagt Stephan Stracke, EU-Berichterstatter der CDU/CSU-Bundestagsfraktion im Gesundheitsausschuss.

Auch der Bundesverband privater Anbieter sozialer Dienste (bpa) spricht sich gegen eine Änderung der Bedingungen zur Pflegeausbildung aus. „Eine erfolgreich abgeschlossene zehnjährige allgemeine Schulbildung muss als Voraussetzung für die Pflegeausbildung gesetzlich garan- tiert bleiben - in Deutschland und in Europa“, mahnt bpa-Präsident Bernd Meurer. Andernfalls würden hierzulande nur noch Abiturienten eine Ausbildung in der Pflege beginnen können. Geeignete Realund Hauptschulabsolventen wären erst mal außen vor. „Das wäre ein fataler Rückschritt und in Zeiten eines dramatischen Fachkräftemangels das grundlegend falsche Signal“, so Meurer.

Der deutsche Berufsverband der Pflegeberufe (DBfK) erhofft sich durch die EUInitiative einen „qualitativen Schub“ für die Weiterentwicklung der Ausbildung. „Diese Änderung ist ein wichtiger Meilenstein in der Professionalisierung der Pflege in Deutschland“, betont die Präsidentin des DBfK, Gertrud Stöcker.

Geänderte Anforderungen soll es nach den Kommissionplänen auch bei den Sprachprüfungen von Ärzten geben. Die derzeit gültige EU-Richtlinie legt fest, dass der Arzt Sprachkenntnisse des Landes nachweisen muss, in das er wechseln will. Dem Revisionsvorschlag zufolge soll eine Überprüfung der Sprachkenntnisse erst nach der Anerkennung der Berufsqualifikation durch das Gastland erfolgen. Es sei Aufgabe des jeweiligen Mitgliedstaates, behördliche Kontrollen der Sprachkenntnisse anzuberaumen, wenn dies erforderlich erscheint, heißt es dazu weiter.

Im EU-Parlament gehen die Meinungen hierzu auseinander. „Ich bin dagegen, Deutsch als Arbeitssprache in der Gesundheitsversorgung zu schwächen“, betont beispielsweise der hessische CDUEuropaabgeordnete und Vizepräsident des Ausschusses für Beschäftigung und Soziales im EP, Thomas Mann. Die Mehrheit der Europaabgeordneten will hingegen keine verpflichtenden Sprachprüfungen. „Patientenberatungen warnen bereits vor zunehmenden Problemen mit mangelhaften Sprachkenntnissen“, so Mann. Sprachprüfungen auf hohem Niveau sollten daher im Medizin-Sektor verpflichtend sein, fordert der CDU-Politiker.

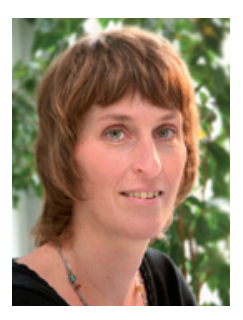

Korrespondenz Petra Spielberg Fachjournalistin für Gesundheits- und Sozialpolitik Wiesbaden/Brüssel Fax. 0611/98818512 p.spielberg@t-online.de 